\title{
RELACIÓN ENTRE LOS SISTEMAS DE CONTABILIDAD Y CONTROL DE GESTIÓN Y LOS SESGOS EN LA EVALUACIÓN Y TOMA DE DECISIONES*
}

\author{
ERNESTO LOPEZ-VALEIRAS**, JACOBO GOMEZ-CONDE*** \& DAVID NARANJO-GIL***** \\ UNIVERSIDAD DE VIGO (ESPAÑA) - UNIVERSIDAD AUTÓNOMA DE MADRID (ESPAÑA) - UNIVERSIDAD PABLO \\ DE OLAVIDE (ESPAÑA)
}

Recibido/ Received/ Recebido: 14/02/15 - Aceptado/ Accepted / Aprovado: 23/10/15

\begin{abstract}
Resumen
Los directivos realizan evaluaciones y toman decisiones que pueden estar sesgadas y, como consecuencia, provocar efectos contrarios a los deseados en el rendimiento organizacional. La identificación de los sesgos y sus causas han sido ampliamente estudiadas en la literatura de contabilidad y control de gestión en las últimas décadas. El objetivo de este trabajo es sintetizar los resultados previos, lo cual permite disponer de una visión más clara y comprensiva del papel de los Sistemas de Contabilidad y Control de Gestión (SCCG) en relación a los sesgos en las decisiones y evaluaciones. Como resultado se obtiene un marco conceptual en el que los SCCG se postulan al mismo tiempo como generadores e inhibidores de sesgo, en función de sus propias características y de las características de los usuarios. Este resultado permite que profesionales (especialmente los controllers) y académicos conozcan mejor los procesos de utilización de la información generada por los SCCG y así actúen en consecuencia.

Palabras clave: Sesgo; Sistemas de contabilidad y control de gestión; Evaluación del rendimiento; Toma de decisiones.

\section{RELATIONSHIP BETWEEN ACCOUNTING SYSTEMS AND CONTROL MANAGEMENT AND THE BIAS IN ASSESSMENT AND DECISION MAKING}

\begin{abstract}
Managers conduct evaluations and make decisions that can be biased and, therefore, cause adverse effects to the desired organizational performance. Identifying the biases and their causes have been widely studied in the accounting literature and management control in recent decades. The aim of this paper is to summarize the previous results, which allows a clear and comprehensive view of the role of Accounting Systems and Management Control (SCCG) in relation to biases in the decisions and assessments. As a result a conceptual framework is obtained in which SCCG are postulated as
\end{abstract}

\footnotetext{
Artículo de investigación científica interinstitucional.

* Profesor del Departamento de Economía Financiera y Contabilidad, Universidad de Vigo. Dirección postal: Facultade de Empresariais e Turismo, Campus as Lagoas, 32004 Ourense (España). Teléfono: 988368 880. Correo electrónico: elvaleiras@uvigo.es.

*** Profesor del Departamento de Contabilidad, Universidad Autónoma de Madrid. Facultad de Ciencias Económicas y Empresariales, 28049 Madrid (España).Correo electrónico: jacobo.gomez@uam.es.

***** Departamento de Economía Financiera y Contabilidad, Universidad Pablo de Olavide. Facultad de Ciencias Empresariales, 41013 Sevilla (España). Correo electrónico: dnargil@upo.es.
} 
both generators and inhibitors of bias, according to its own characteristics and the characteristics of users. This result allows professionals (especially controllers) and academicians to know better processes using the information generated by SCCG and so act accordingly.

Keywords: Bias; Accounting systems and management control; Performance evaluation; Decision making.

\title{
RELAÇÃO ENTRE OS SISTEMAS DE CONTABILIDADE E DE CONTROLE DE GERENCIAMENTO E DAS DISTORÇÕES NA AVALIAÇÃO E NA TOMADA DE DECISÕES
}

\begin{abstract}
Resumo
Os diretores realizam avaliações e tomam decisões que podem estar equivocadas e, como consequência, provocar efeitos contrários aos desejados no rendimento organizacional. A identificação das distorções e suas causas têm sido amplamente estudadas na literatura de contabilidade e de controle de gerenciamento nas últimas décadas. O objetivo deste trabalho é sintetizar os resultados prévios, o qual permite dispor de uma visão mais clara e compreensiva do papel dos Sistemas de Contabilidade e de Controle de Gerenciamento (SCCG) em relação às distorções nas decisões e avaliações. Como resultado se obtém um marco conceitual no qual os SCCG se apresentam ao mesmo tempo como geradores e inibidores de distorção, em função de suas próprias características $e$ das características dos usuários. Este resultado permite que profissionais (especialmente os controllers) e acadêmicos conheçam melhor os processos de utilização da informação gerada pelos SCCG e assim ajam de maneira consequente com isto.

Palavras chave: Distorção; Sistemas de contabilidade e de controle de gerenciamento; Avaliação do rendimento; Tomada de decisões.

López-Valeiras, E., Gómez-Conde J. \& Naranjo-Gil, D. (2016). Relación entre los sistemas de contabilidad y control de gestión y los sesgos en la evaluación y toma de decisiones. En: Revista de la Facultad de Ciencias Económicas de la Universidad Militar Nueva Granada. rev.fac.cienc.econ, XXIV Nº1, DOI: http://dx.doi.org/10.18359/rfce.1618.
\end{abstract}

JEL: M15, M41, M49, D81.

\section{Introducción}

Los trabajadores dentro de las empresas pueden actuar de forma egoísta, es decir, poniendo sus propios objetivos e intereses por delante de los objetivos y metas de la organización. Así, pueden surgir comportamientos disfuncionales en las organizaciones (Griffin \& Lopez, 2005; Probst et al., 1999). Los comportamientos disfuncionales son aquéllos que realizan los empleados con consecuencias negativas para el resto de sus compañeros o para los intereses generales de su empresa (Griffin \& Lopez, 2005). Estos comportamientos disfuncionales influyen directa y negativamente en el ambiente y productividad de la empresa.
Existen numerosos comportamientos disfuncionales, entre los que más se han destacado en la literatura de organización y gestión de empresas encontramos: el bajo compromiso con los objetivos y metas de la empresa, el aumento del conflicto en la realización de las actividades internas de la organización, y la reducción del esfuerzo en la consecución y realización de las tareas (Maas \& Van Rinsum, 2013; Roman, 2009). Por ello se hace necesario que la dirección de las organizaciones usen los mecanismos apropiados para reducir o eliminar este tipo de comportamientos disfuncionales. Entre los sistemas o mecanismos más adecuados para este fin se encuentran los sistemas de contabilidad y control de gestión. 
Los sistemas de contabilidad y control de gestión (en adelante SCCG) pueden ser usados por la dirección de las empresas para incentivar y motivar a sus empleados a comportarse y actuar en pro de los objetivos de la organización (Naranjo-Gil \& Hartmann, 2007). Podemos decir que los SCCG se encargan de suministrar la información de gestión y los incentivos para (1) asegurar un comportamiento apropiado de los empleados de una organización (2) crear y supervisar las condiciones que incentiven a los empleados a trabajar por el beneficio de la empresa, y (3) facilitar la coordinación de las tareas productivas (Roman, 2009; Rowe, 2004). Por tanto, los SCCG deben diseñarse y también usarse de forma adecuada para que puedan facilitar la reducción de los comportamientos disfuncionales en las organizaciones. Por tanto, se hace fundamental entender cómo diferentes diseños y usos de los SCCG influyen en los comportamientos disfuncionales de los empleados en las organizaciones.

Una línea de investigación en contabilidad de gestión analiza esta relación centrándose en el estudio de los sesgos existentes en la evaluación y toma de decisiones a partir de información contable. Es decir, un factor que promueve comportamientos disfuncionales como los anteriormente indicados es la presencia de sesgos en evaluaciones de rendimiento y toma de decisiones basadas en información suministrada por los $\mathrm{SCCG}^{1}$. Estos sesgos tienen consecuencias directas e indirectas. Entre las primeras se encuentra, por ejemplo, el coste económico originado por el inflado artificial de las evaluaciones ligadas a incentivos o por la posible desmotivación causada por evaluaciones sesgadas (Moers, 2005). Entre las implicaciones indirectas está, por ejemplo, la dificultad de tomar decisiones relacionadas con el personal, caso de la asignación de puestos (Moers, 2005). Específicamente Golman \& Bhatia (2012) sugieren que los sesgos de lenidad y tendencia central producidos durante evaluaciones del rendimiento a partir de la información suministrada

La literatura contable también reconoce la existencia de sesgos con efectos positivos como aquellos en los que los directivos realizan evaluaciones sesgadas para ayudar a los trabajadores, por motivos relacionados con la formación de trabajadores o para evitar conflictos entre empleados (para más detalles ver Golman \& Bhatia, 2012). por SCCG producen un descenso de los salarios de los trabajadores y, por tanto, afectan su rendimiento y al rendimiento de la empresa.

Wyatt (1986) mantiene que la toma de decisiones debe ser lo más neutral posible, centrándose en los aspectos relevantes y descartando otros que puedan sesgar las conclusiones alcanzadas. Sin embargo, existe un consenso generalizado en la literatura sobre la falta de neutralidad asociada a las decisiones y evaluaciones basadas en información suministradas por los SCCG. En esta línea existen multitud de trabajos que han tratado de aumentar el conocimiento sobre la relación SCCG-sesgo utilizando diversas metodologías, terminologías, marcos teóricos, niveles de análisis, tipos de SCCG, variables explicativas y variables explicadas. Además, paralelamente, se sugieren diversos factores que pueden contribuir a la reducción de estos sesgos y, por ende, de los comportamientos disfuncionalidades derivados de ellos. Por todo ello, los resultados obtenidos son diversos $y$, sin embargo, hasta la fecha no existe ningún trabajo integrador que permita mejorar el conocimiento existente en este ámbito.

El objetivo de este estudio es sintetizar los resultados obtenidos en los trabajos previos para identificar la dirección, intensidad y variables relacionadas con la existencia de sesgos en el diseño y utilización de SCCG. Para cumplir con el objetivo propuesto se realiza una revisión sistemática que sigue una rigurosa metodología para la búsqueda, selección y análisis de los trabajos. Como resultado se obtiene un marco conceptual en el que los SCCG se postulan al mismo tiempo como generadores e inhibidores de sesgo, en función de sus propias características (por ejemplo el tipo de sistema que genera la información o el grado de subjetividad implícita) y de las características de los usuarios (por ejemplo el sentimiento de responsabilidad, el sentimiento afectivo, los intereses individuales o las limitaciones de procesamiento de información).

El resto del artículo está estructurado del siguiente modo. En el segundo capítulo se justifica brevemente la relevancia que tienen la generación de sesgos a partir de la información suministrada por los SCCG, así como una breve descripción de algunos de los 
sesgos que más han captado la atención de los investigadores en contabilidad de gestión. El tercer capítulo presenta la metodología seguida en la realización de este estudio. Los resultados obtenidos desglosados por bloques y el marco conceptual basado en ellos ocupan el cuarto capítulo. Por último se detallan las principales conclusiones obtenidas.

\section{Sistemas de contabilidad y control de gestión, sesgo y disfuncionalidad}

En la literatura contable sesgo es el efecto que ejerce sobre una evaluación/decisión la tendencia que tiene un usuario/diseñador de SCCG a decidir a favor de una opción en detrimento de otra $^{2}$ (Blocher et al., 1986). En este sentido Waller et al., (1999) analizan las aproximaciones dadas por economistas y psicólogos respecto a la racionalidad en la toma de decisiones. La literatura económica se basa en que los individuos toman decisiones racionales que maximizan la utilidad percibida. Por su parte la literatura de psicología sugiere que la presencia de sesgos en la toma de decisiones viene dado por la forma en la que el ser humano procesa la información. Con el objetivo de "ahorrar" recursos cognitivos los individuos utilizan atajos mentales ${ }^{3}$ o simplificaciones de la realidad que inevitablemente conducen a comportamientos y decisiones sesgadas.

Unas de las funciones fundamentales de los SCCG es suministrar información para (1) el desarrollo de los procesos de planificación, control y evaluación del rendimiento y (2) para la toma de decisiones (Drury, 2012). Entre estas últimas se encuentran por ejemplo el establecimiento de precios, la aceptación o rechazo de un pedido o la subcontratación de actividades. Tanto la toma de decisiones como los procesos de planificación, control y evaluación del rendimiento pueden sufrir sesgos.

2 En este estudio se distinguen los conceptos de sesgo y precisión en las decisiones o evaluaciones basadas en SCCG. La precisión captura la "habilidad que tiene un individuo de utilizar reglas de decisión correcta y consistentemente para clasificar las observaciones de dos alternativas" (Blocher et al., 1986, p. 459).

3 También conocidas como heurísticas.

\subsection{Sesgos en planificación, control y evaluación del rendimiento}

Dos de los sesgos más habituales son los relacionados con la inflación y la compresión en las evaluaciones del rendimiento. Entre los de inflación, el sesgo de lenidad ${ }^{4}$ (o indulgencia) consiste en realizar una evaluación más favorable de la que en realidad correspondería, por lo cual se aprecia que demasiadas evaluaciones se encuentran por encima de la media. Una medida habitual del sesgo de lenidad es la diferencia entre los valores medios otorgados a la población objeto de evaluación y los valores reales medios en esa determinada competencia (Golman \& Bathia, 2012). Entre los de compresión, el sesgo de tendencia central ${ }^{5}$ reside en realización de evaluaciones que evitan las puntuaciones extremas, es decir, la tendencia a la utilización de valores medios. Esto supone la existencia de poca variación entre las valoraciones realizadas. En este caso la varianza de la distribución de las valoraciones realizadas sobre una determinada competencia es menor que la de la distribución real (Golman \& Bathia, 2012). En un contexto de evaluaciones de rendimiento en empleados, una de las consecuencias de la existencia simultánea de sesgo de lenidad y de tendencia central es que hay una insuficiente diferenciación entre empleados (Moers, 2005) y los empleados con menores competencias obtienen las valoraciones más infladas (Golman \& Bathia, 2012).

Otros dos tipos de sesgos en los procesos de evaluación del rendimiento son los relacionados con la atención selectiva por parte del usuario de la información suministrada por los SCCG: sesgo de actor-observador y el sesgo de correspondencia. El sesgo de actor-observador viene dado por la distinta consideración del peso de factores del entorno en el rendimiento. En el marco de los SCCG, los evaluados (actores) tienden a otorgar más peso a los factores contextuales entre las causas de su rendimiento que el que le otorgan los evaluadores (observadores). La calidad de la estrategia organizativa es un ejemplo de factor contextual (Wong-On-Wing et al., 2007). Al igual que el sesgo actor-observador,

\footnotetext{
Leniency bias en inglés.

Centrality bias en inglés.
} 
el sesgo de correspondencia también ignora factores contextuales como posible causa de un determinado rendimiento. Sin embargo el sesgo surge en el momento que evaluador realiza inferencias únicamente sobre las disposiciones del evaluado obviando la situación en la que se generó la acción. Así, por ejemplo, el rendimiento aparentemente bajo de un individuo será evaluado más negativamente (sesgo) por un evaluador que ignore el papel de la estrategia en los resultados que por un evaluador que sí lo tenga en cuenta.

Un sesgo habitual en el proceso de planificación es la existencia de holguras, por ejemplo, en la confección de presupuestos siguiendo una metodología participativa se establecen objetivos por debajo de lo que sería óptimo debido a un comportamiento egoísta de alguno de los agentes intervinientes (Waller, 1988).

\subsection{Sesgos en la toma de decisiones}

La compra o fusión de otras empresas, la introducción de nuevas líneas de productos, la implementación de nuevos procesos productivos o la realización de cambios en la capacidad productiva son decisiones complejas y de carácter estratégico habituales en la gestión de empresas. El riesgo e incertidumbre existentes pueden dar lugar a una sobrevaloración de los indicadores financieros y por tanto a la toma de decisiones sesgadas hacia decisiones más a corto plazo con beneficios cuantificables objetivamente (Alkaraan \& Northcott, 2006).

Esta situación también puede estar presente en decisiones de carácter más operativo. Un potencial sesgo se produce en el caso de los auditores internos que deben decidir sobre el riesgo de los informes. En este caso el sesgo en la decisión es "una medida sobre la propensión intrínseca de responder que un informe es de riesgo alto o bajo, independientemente del contenido de cualquiera de los informes" (Blocher et al., 1986, p. 461). En esta misma línea se encuentra la existencia de sesgos en toma decisiones relacionadas con el establecimiento de precios de transferencia. Así, las partes implicadas en la negociación asignarán mayor peso a las informaciones que más se acercan a sus expectativas e intereses (Chang et al., 2008). Otra decisión que puede contener sesgo es la continuidad o abandono de un proyecto (Harrell \& Harrison, 1994).

\section{Metodología}

Para la revisión de la literatura se utilizó la metodología seguida por Franco-Santos et al. (2012). Inicialmente se formó una base de artículos clave sobre el tema para realizar un estudio de alcance. Los artículos utilizados en esta fase fueron aquellos que Franco-Santos et al. (2012) clasificaron en el apartado de "judgement biases". Esta lista contiene los siguientes artículos: Libby et al. (2004); Kaplan \& Wisner (2009); Lipe \& Salterio (2000, 2002); Tayler (2010); Wong-on-Wing et al. (2007); Liedtka et al. (2008); Cardinaels \& Van Veen-Dirks (2010); Banker et al. (2004); Moers (2005); Roberts et al. (2004); Schiff \& Hoffman (1996); Dilla \& Steinbart (2005).

Como resultado se identificaron las siguientes palabras clave para una posterior búsqueda de trabajos relevantes: "sesgo"; "medición del rendimiento"; y "control de gestión"6. Teniendo en cuenta el alcance de nuestro estudio y siguiendo la propuesta de Franco-Santos et al. (2012) se seleccionaron 5 revistas contables ${ }^{7}$ reconocidas por publicar trabajos de alta calidad: Accounting, Organization and Society; The Accounting Review; Management Accounting Research; Behavioral Research in Accounting; y British Accounting Review. La revisión abarca el período comprendido entre 1972 (por ser la fecha de publicación del trabajo seminal de Hopwood (1972) en el que trata el papel de la información contable en la evaluación de rendimiento) y diciembre de 2014.

La búsqueda se realizó utilizando las palabras clave indicadas anteriormente en los buscadores vinculados desde las páginas oficiales de las revistas seleccionadas. La lectura de los resúmenes permitió realizar la descarga únicamente de los artículos que cumplían con los requisitos marcados para nuestro

La búsqueda se realizó con los correspondientes términos en inglés: "bias"; "performance meas"; y "management control". Franco-Santos et al. (2012) incluyen en esta fase también 5 revistas de gestión y estrategia y otras 5 revistas de operaciones. Decidimos no incluir estas revistas en nuestro proceso debido a las características y alcance de nuestro trabajo, más específico y focalizado que el de Franco-Santos et al. (2012). 
estudio. En este proceso también se identificaron otros estudios relevantes publicados en otras revistas mediante un análisis de referencias cruzadas.

\section{Resultados}

La búsqueda bibliográfica y posterior filtrado concluyó con la identificación de 24 artículos que cumplían los requisitos propuestos. Los resultados se organizaron en las Tablas 1, 2 y 3 . Como resultado se obtuvo el marco conceptual (Figura 1) en el que se presentan los factores que intervienen favoreciendo o inhibiendo la presencia de sesgos en los juicios tomados a partir de la información generada por los SCCG. Los resultados para cada uno de estos factores se presentan a continuación.

Figura 1. Marco conceptual sobre los factores que anteceden a los sesgos en evaluaciones y toma de decisiones.

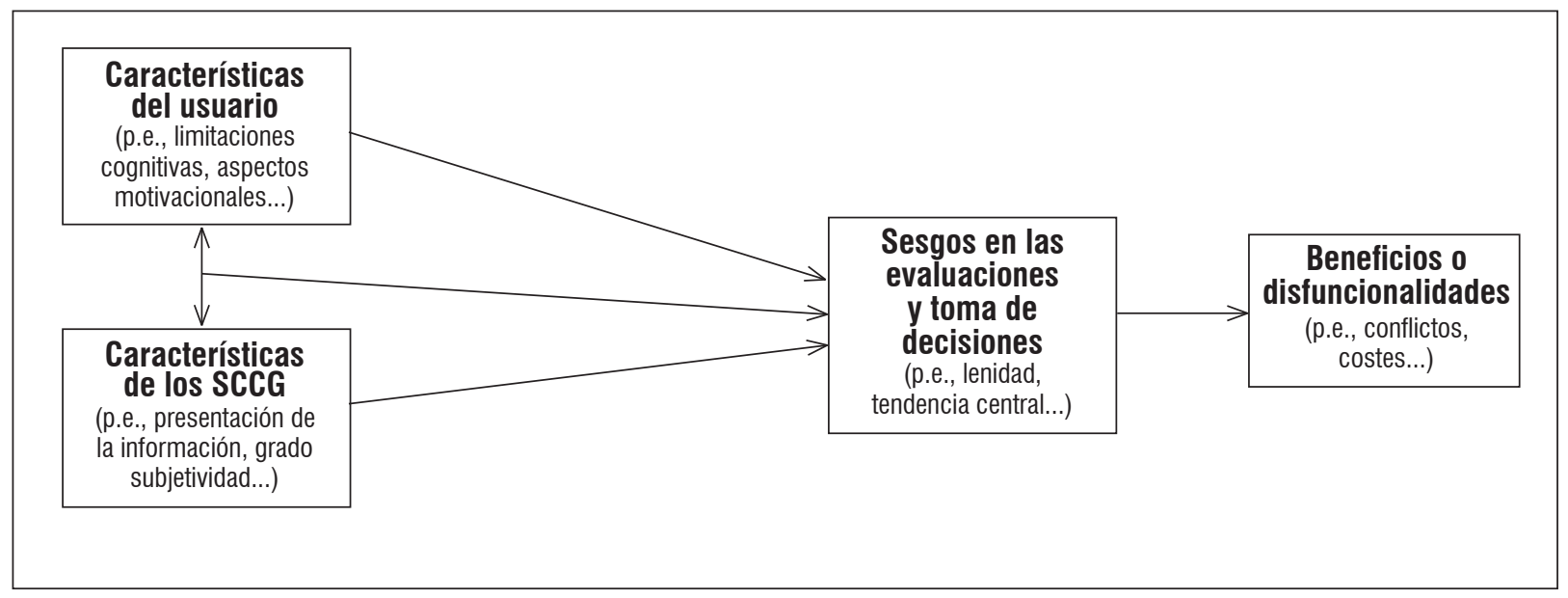

Fuente: Elaboración propia.

\subsection{Características del SCCG}

La revisión de la literatura muestra que los SCCG pueden desempeñar un doble papel. Por un lado determinados diseños de los SCCG pueden ser fuentes directas de generación de juicios sesgados. Por otro lado, los SCCG pueden contribuir a reducir (o incrementar) los sesgos en los juicios derivados de las limitaciones cognitivas o intereses particulares de los usuarios de la información contable. Así, un tema de estudio clásico en la literatura de contabilidad de gestión es el análisis del efecto que la utilización de SCCG alternativos tiene sobre la toma de decisiones. En este sentido, Waller et al. (1999) dan continuidad a una línea de investigación centrada en analizar el sesgo generado en el establecimiento de precios de venta en función del método de cálculo de costes en el que se basa dicha decisión. Trabajos previos habían sugerido que el establecimiento de precio basado en coste de absorción eran significativamente más altos que cuando se utilizaba un sistema de costes variables. Waller et al., (1999) evidenciaron que las condiciones del mercado (en especial la demanda) juega un papel fundamental en esa relación eliminado el sesgo en el establecimiento de precio generado por los métodos alternativos de cálculo de costes.

La presencia de imprecisiones o errores en la información suministrada ha sido considerada también como un factor que puede conducir a sesgos $\mathrm{y}$, por ende, a disfuncionalidades. En este sentido Alkaraan \& Northcott (2006) destacan como ejemplos la existencia de inexactitudes en las estimaciones de las variables, la consideración de ratios impropios y la omisión de datos importantes.

Otro aspecto de los SCCG que ha sido ampliamente estudiado es el formato de presentación de la información por su influencia sobre la capacidad de asi- 
milación de la información que desarrollan los usuarios. Entre otros destacan las posibles presentaciones y formatos de indicadores en el cuadro de mando integral (Roberts et al., 2004; Lipe \& Salterio, 2000, 2002; Cardinaels \& van Veen-Dirks, 2010), el número de perspectivas incluidas en el cuadro de mando integral (Kaplan \& Wisner, 2009), el enmarcado de la información (Chang et al., 2008) o la diversidad de medidas utilizadas (Moers, 2005).

La evaluación del desempeño a través de indicadores subjetivos es una práctica valiosa y habitual en las empresas, especialmente cuando los indicadores objetivos son difíciles de obtener, son costosos o son imprecisos (Golman \& Bhatia, 2012) o la decisión a tomar es de carácter estratégico (Alkaraan \& Northcott, 2006). También se ha defendido la utilización combinada de indicadores objetivos y subjetivos para el reparto de incentivos (Moers, 2005). La subjetividad puede introducirse por ejemplo otorgando discrecionalidad a los decisores de otorgar pesos específicos a los distintos indicadores del SCCG ligados con los incentivos (Burney et al., 2009). También la presencia de indicadores cualitativos da lugar a subjetividad en los juicios realizados por los evaluadores (Moers, 2005). Sin embargo trabajos en distintas áreas de conocimiento demuestran empíricamente que la subjetividad da lugar a discrecionalidad que puede producir efectos indeseables entre los que se encuentra la generación de sesgos (p. ej. Moers, 2005; Ittner et al., 2003; Prendergast \& Topel, 1993).

A modo de resumen, la Tabla 1 recoge los trabajos en los que se incluyen variables relacionadas con las características de los SCCG, y se muestra su vinculación con los sesgos. Al igual que en las Tablas 2 y 3 , los modelos propuestos originalmente en estos trabajos pueden ser más amplios, incluyendo otras variables que no están recogidas en esta tabla.

Tabla 1. Características de los SCCG y sesgos

\begin{tabular}{|c|c|c|c|}
\hline Referencia & Variable(s) independiente(s) & Variable(s) moderadora(s) & Variable(s) dependiente(s) (sesgo) \\
\hline \multirow{2}{*}{ Burney et al. (2009) } & *Validez técnica de los SCCG $(+)$ & & \multirow{2}{*}{-Justicia } \\
\hline & *Grado de relación estratégica causal (+) & & \\
\hline Waller et al. (1999) & *Tipo de sistema de costes utilizado (n.s.) & -Condiciones de la demanda & -Sesgo en el establecimiento de precios \\
\hline Blocher et al. (1986) & *Formato del informe (n.s) & - Complejidad de la tarea $(+)$ & - Sesgo en la toma de decisión \\
\hline \multirow{2}{*}{ Waller (1988) } & *Sistema convencional de negociación (+) & \multirow{2}{*}{-Aversión al riesgo (-) } & \multirow{2}{*}{-Holguras en presupuestos } \\
\hline & *Sistema inducidor de la verdad (-) & & \\
\hline \multirow{2}{*}{ Chang et al. (2008) } & -Atención selectiva $(+)$ & \multirow{2}{*}{$\begin{array}{l}\text { *Enmarcado de la información } \\
\text { contable }(+)\end{array}$} & \multirow{2}{*}{-Diferencias en precios de transferencia } \\
\hline & -Factores sociales $(+)$ & & \\
\hline Cardinaels \& van Veen-Dirks (2010) & ${ }^{*}$ Formato BSC $(+)$ & & -Peso de los indicadores financieros \\
\hline Lipe \& Salterio (2002) & ${ }^{*}$ Formato BSC $(+)$ & -Ruido en las medidas (+) & -Sesgo de centralidad \\
\hline \multirow[t]{2}{*}{ Moers (2005) } & ${ }^{*}$ Diversidad de medidas $(+)$ & & -Sesgo de comprensión \\
\hline & *Subjetividad (+) & & -Sesgo de lenidad \\
\hline Harwood et al. (1991) & $\begin{array}{l}\text { *Enmarcado de la información contable } \\
(+)\end{array}$ & $\begin{array}{l}\text {-Formación (n.s.) } \\
\text {-Experiencia presente (n.s.) } \\
\text {-Experiencia pasada (n.s.) }\end{array}$ & -Sesgo en la toma de decisiones \\
\hline Kaplan et al. (2012) & ${ }^{*}$ Formato BSC $(+)$ & & -Sesgo en las evaluaciones \\
\hline Roberts et al. (2004) & $\begin{array}{l}\text { *Formato BSC (medidas comunes y únicas } \\
\text { para departamentos) }(+)\end{array}$ & & -Sesgo en la compensación \\
\hline
\end{tabular}




\begin{tabular}{|c|c|c|c|}
\hline Referencia & Variable(s) independiente(s) & Variable(s) moderadora(s) & Variable(s) dependiente(s) (sesgo) \\
\hline Libby et al. (2004) & $\begin{array}{l}\text {-Justificación de la decisión. Rendición de } \\
\text { cuentas }(+) \\
{ }^{\star} \text { Report de terceras partes }(+)\end{array}$ & & -Sesgo en la evaluación del rendimiento \\
\hline Cardinaels \& Labro (2008) & $\begin{array}{l}\text { *Agregación de medidas }(+) \\
{ }^{*} \text { Notificaciones de evolución }(+)\end{array}$ & & -Sesgo en la estimación de tiempos (evaluación) \\
\hline Dilla \& Steinbart (2005) & $\begin{array}{l}\text { *Formato BSC (medidas comunes y únicas } \\
\text { para departamentos) }(+)\end{array}$ & -Formación $(+)$ & -Sesgo en rendimiento y asignación de bonus \\
\hline Kaplan \& Wisner (2009) & $\begin{array}{l}\text { *Formato BSC (inclusión quinta perspec- } \\
\text { tiva) }\end{array}$ & $\begin{array}{l}\text {-Comunicación con los gesto- } \\
\text { res }(+)\end{array}$ & -Sesgo en evaluación del rendimiento. \\
\hline Lipe \& Salterio (2000) & $\begin{array}{l}\text { *Formato BSC (medidas comunes y únicas } \\
\text { para departamentos) }(+)\end{array}$ & & -Sesgo en evaluación del rendimiento. \\
\hline Schiff \& Hoffman (1996) & $\begin{array}{l}\text { *Información financiera y no financiera } \\
(+/-)\end{array}$ & & $\begin{array}{l}\text {-Sesgo en evaluación del rendimiento de de- } \\
\text { partamento/managers }\end{array}$ \\
\hline Banker et al. (2004) & $\begin{array}{l}{ }^{*} \text { Formato BSC (medidas comunes y únicas } \\
\text { para departamentos) }(+)\end{array}$ & & -Sesgo en evaluación del rendimiento. \\
\hline
\end{tabular}

* Variables de características de los SCCG.

Fuente: Elaboración propia.

\subsection{Características del usuario}

Otra de las razones que explica la existencia de sesgos en las evaluaciones y toma de decisiones basadas en información contable es la propia existencia de sesgos en los usuarios de dicha información. Es decir, los sesgos de los individuos se transmiten a las decisiones y evaluaciones. Los sesgos en los individuos pueden deberse tanto a aspectos motivacionales como a sus propias limitaciones cognitivas.

Al igual que en el caso de las características de los SCCG, la revisión de la literatura muestra que las características de los usuarios de la información contable pueden desempeñar un doble papel. Por un lado las características de los usuarios pueden ser fuentes directas de generación de juicios sesgados. Por otro lado, también pueden contribuir a reducir (o incrementar) los sesgos en los juicios derivados de las características de los SCCG. Las características de los usuarios se agrupan en limitaciones cognitivas y aspectos motivacionales.

\subsubsection{Limitaciones cognitivas}

Los potenciales beneficios obtenidos de la utilización de la información generada por los SCCG pueden verse reducidos debido a las limitaciones cognitivas de los usuarios de dicha información (Lipe \& Salterio, 2000, 2002). Golman \& Bathia (2012) parten de la premisa de que los directivos están motivados a realizar evaluaciones precisas, pero al mismo tiempo también tienen sentimientos altruistas respecto a las personas evaluadas. Así, una de las limitaciones cognitivas que fue identificada como fuente de sesgo es la atención selectiva a la información suministrada por los SCCG. Los seres humanos son incapaces de procesar más de nueve detalles de información al mismo tiempo (Lipe \& Salterio, 2002). Por este motivo, por ejemplo, el papel desempeñado por los usuarios condicionará la información que consideran relevante, es decir, un usuario puede ignorar indicadores a los cuales otros usuarios les otorgan gran importancia (Wong-On-Wing et al., 2007; Chang et al., 2008). En esta misma línea se encuentra la atención selectiva propiciada por la implicación de los usuarios en 
la cuestión sobre la que hay que tomar la decisión o realizar la evaluación (Tayler, 2010). Por el contrario, las diferencias (sesgos) en juicios basados en la misma información contable pueden mitigarse si los individuos desarrollan pensamiento comprensivo ${ }^{8}$ (Butler \& Ghosh, 2015). Se entiende como la habilidad que pueden desarrollar los seres humanos de pensar sobre múltiples caminos, ramificaciones y alternativas.

Otros factores cognitivos que han sido cumplidamente estudiados en la literatura por su capacidad de explicar juicios realizados al margen de la racionalidad económica son aquellos relacionados con cuestiones sociales. Es decir, las percepciones que tienen los usuarios de la información contable sobre las personas involucradas o relacionadas con la información o los juicios que se vas a derivar de ella. Por ejemplo, los sentimientos afectivos respecto a la persona evaluada, la percepción de desigualdad, la percepción de los objetivos de las otros agentes implicados (Chang et al., 2008).

La Tabla 2 recoge los trabajos obtenidos de la revisión de la literatura en los que se incluyen variables relacionadas con las limitaciones cognitivas, y se muestra su vinculación con los sesgos.

Tabla 2. Limitaciones cognitivas y sesgos

\begin{tabular}{|c|c|c|c|}
\hline Referencia & Variable(s) independiente(s) & Variable(s) moderadora(s) & Variable(s) dependiente(s) (sesgo(s)) \\
\hline \multirow{2}{*}{ Wong-on-Wing et al. (2009) } & \multirow{2}{*}{ *Atención selectiva } & \multirow{2}{*}{$\begin{array}{l}\text {-Conciencia de la importancia de la } \\
\text { estrategia }\end{array}$} & -Actor-observador \\
\hline & & & -Correspondencia \\
\hline \multirow{2}{*}{ Waller (1988) } & -Sistema convencional de negociación (+) & \multirow{2}{*}{ *Aversión al riesgo (-) } & \multirow{2}{*}{-Holguras en presupuestos } \\
\hline & -Sistema inducidor de la verdad (-) & & \\
\hline \multirow{2}{*}{ Chang et al. (2008) } & *Atención selectiva (+) & \multirow{2}{*}{$\begin{array}{l}\text {-Enmarcado de la información con- } \\
\text { table }(+)\end{array}$} & \multirow{2}{*}{-Diferencias en precios de transferencia } \\
\hline & ${ }^{*}$ Factores sociales $(+)$ & & \\
\hline Liedka et al. (2008) & *Intolerancia a ambigüedad (+) & & -Sesgo en la evaluación del rendimiento \\
\hline
\end{tabular}

* Variables de aspectos motivacionales.

Fuente: Elaboración propia.

\subsubsection{Aspectos motivacionales}

Este tipo de factores hacen referencia a la tendencia que tienen los individuos a considerar que la opción más justa es la que ellos defienden (Chang et al., 2008). Un directivo puede preferir, en presencia de incertidumbre, hacer evaluaciones que eviten errores de evaluación. Por ejemplo, en el caso de auditores internos que tienen que decidir si un determinado informe representa un riesgo alto o bajo de auditoría un error sería decir riesgo bajo cuando es bajo, es decir, una falta (en inglés miss). El otro error sería decir alto cuando realmente es bajo, es decir, una falsa alarma. El coste asociado a cada uno de es- tos dos errores influenciará la tendencia a tomar una determinada decisión (sesgo) por parte del auditor interno (Blocher et al., 1986).

Otro factor en línea con los costes asociados a los errores de mala clasificación se encuentra las preferencias de equidad. Éstas suponen un comportamiento egoísta del evaluador que conducirá a un sesgo de sobreevaluación (p.ej. sesgo de lenidad) sobre las competencias reales de la persona evaluada (Golman $\&$ Bathia, 2012). Uno de estos factores es la asimetría en la aversión a realizar evaluaciones inmerecidamente altas e inmerecidamente bajas. La Tabla 3 recoge los obtenidos de la revisión de la literatura.

\footnotetext{
8 Comprehensive thinking ability (Butler \& Gosh, 2015).
} 
Tabla 3. Aspectos motivacionales y sesgos

\begin{tabular}{|c|c|c|c|}
\hline Referencia & Variable(s) independiente(s) & Variable(s) moderadora(s) & Variable(s) dependiente(s) (sesgo) \\
\hline \multirow{2}{*}{ Harrell \& Harrison (1994) } & *Incentivo para eludir $(+)$ & & \multirow{2}{*}{-Inflación en las evaluaciones } \\
\hline & *Posesión de información privada (+) & & \\
\hline Harwood et al. (1991) & -Enmarcado de la información contable (+) & $\begin{array}{l}\text { *Formación (n.s.) } \\
\text { *Experiencia presente (n.s.) } \\
\text { *Experiencia pasada (n.s.) }\end{array}$ & -Sesgo en la toma de decisiones \\
\hline Bol (2011) & $\begin{array}{l}\text { *Relaciones fuertes entre empleado y direc- } \\
\text { tivo }(+) \\
{ }^{\star} \text { Coste de búsqueda de la información }(+)\end{array}$ & & $\begin{array}{l}\text {-Sesgo de lenidad } \\
\text {-Sesgo de centralidad }\end{array}$ \\
\hline Libby et al. (2004) & $\begin{array}{l}\text { *Justificación de la decisión. Rendición de } \\
\text { cuentas }\end{array}$ & & -Sesgo en la evaluación del rendimiento \\
\hline Tayler (2010) & $\begin{array}{l}\text { *Participación en la selección de iniciativas } \\
\text { estratégicas }(+)\end{array}$ & $\begin{array}{l}\text {-Énfasis en la perspectiva fi- } \\
\text { nanciera }(+)\end{array}$ & $\begin{array}{l}\text {-Sesgo en la evaluación del éxito de la } \\
\text { iniciativa estratégica }\end{array}$ \\
\hline
\end{tabular}

* Variables de aspectos motivacionales.

Fuente: Elaboración propia.

\section{Conclusiones}

La presencia de sesgos en la evaluación y toma de decisiones a partir de información contable es uno de los retos a los que se enfrentan los directivos a la hora de diseñar y utilizar un SCCG. Entre otras consecuencias se encuentran la reducción del valor de la información suministrada y de las decisiones tomadas; reducción de la motivación de los trabajadores y de la productividad organizativa (Golman \& Bhatia, 2012). En el ámbito académico existen múltiples y diferenciadas aproximaciones que han dedicado un esfuerzo significativo a tratar de explicar esta relación.

El objetivo de este trabajo es contribuir a la literatura de control de gestión aportando un marco conceptual que permita una mejor comprensión de la relación entre los SCCG y la existencia de sesgos en las evaluaciones y toma de decisiones. Para ello, tras una revisión sistemática, se identificaron 24 artículos a partir de los cuales se diseñó el marco conceptual. Los resultamos sugieren que los SCCG pueden actuar al mismo tiempo como generadores $e$ inhibidores de sesgo, en función de sus propias características y de las características de los usuarios de los SCCG.
La literatura previa analizada se centra fundamentalmente en explicar la relación entre los SCCG y los sesgos en la evaluación y toma de decisiones desde la perspectiva del usuario dejando de lado el papel que desempeñan los diseñadores de las herramientas. Es necesario tener en cuenta que, en muchos casos, diseñadores y usuarios no coinciden en la misma persona. Una de las tareas de los responsables del diseño de los SCCG es la de, en la medida de lo posible, proveer de información neutral (imparcial) a las personas que deben tomar decisiones en la organización (Solomons, 1991).

La principal preocupación a la hora de generar información contable debe ser la relevancia y la confiabilidad de la misma, y no el efecto que pueda tener sobre intereses particulares. En este sentido, FASB (1980, p. 25) indica que "para ser neutral, la información contable debe informar de la actividad económica lo más fielmente posible, sin colorear la imagen que se comunica con el propósito de influir en el comportamiento de alguna dirección en particular". Sin embargo, la neutralidad absoluta en la elaboración de información es difícilmente alcanzable ya que el preparador de la información (o el diseñador de los SCCG) ejercerá determinado sesgo que se transmitirán a dicha información (Tinker, 1995). 
Por tanto, la base para el diseño y el desarrollo de un SCCG es establecer los requisitos de información: identificación de la información que debe generar y su disponibilidad, formato en el que será presentada la información, y los usuarios (Boland, 1979; Blocher et al., 1986). Sin embargo estas tareas no son sencillas debido al elevado número de variables que intervienen. Según Boland (1979), este análisis puede ser una fuente potencial de sesgo junto con el sesgo derivado de la comprensión que el propio diseñador tenga de la causalidad de los procesos propios de cada organización y la intención de uso que el diseñador conciba para la herramienta. En línea con lo sugerido por Franco-Santos et al., (2012), futuros trabajos deberían prestar más atención al papel desempeñado por los diseñadores de los SCCG ya que tanto sus limitaciones cognitivas como aspectos motivacionales condicionarán el diseño de las propias herramientas, que a su vez pueden ser causa u obstáculo de evaluaciones y decisiones sesgadas.

Este trabajo tiene una serie de limitaciones que deben ser consideradas. En primer lugar la revisión de la literatura restringe a 5 revistas. Para evitar la omisión de otros estudios relevantes, futuros trabajos deberían ampliar la búsqueda a otras revistas contables de impacto e incluir otros trabajos citados en los manuscritos seleccionados. En segundo lugar, el área de revisión se ciñe a la literatura contable. Futuros trabajos deberían incluir revistas de otros ámbitos como el de gestión de empresas y operaciones.

\section{Referencias}

Alkaraan, F. \& Northcott, D. (2006) "Strategic capital investment decision-making: A role for emergent analysis tools?: A study of practice in large UK manufacturing companies". En: The British Accounting Review, 38(2): 149-173.

Banker, R.D., Chang, H.S. \& Pizzini, M.J. (2004) "The balanced scorecard: judgmental effects of performance measures linked to strategy". En: The Accounting Review, 79(1): 1-23.

Blocher, E., Moffie, R.P. \& Zmud, R.W. (1986) "Report format and task complexity: Interaction in risk judgments". En: Accounting, Organizations and Society, 11(6): 457-470.

Bol, J.C. (2011) "The determinants and performance effects of managers' performance evaluation biases". En: The Accounting Review, 86(5): 1549-1575.

Boland Jr., R.J. (1979) "Control, causality and information system requirements". En: Accounting, Organizations and Society, 4(4): 259-272.

Burney, L.L., Henle, C.A. \& Widener, S.K. (2009) "A path model examining the relations among strategic performance mea- surement system characteristics, organizational justice, and extra- and in-role performance". En: Accounting, Organizations and Society, 34(3-4): 305-321.

Butler, S.A. \& Ghosh, D. (2015) "Individual differences in managerial accounting judgments and decision making". En: The British Accounting Review, 47,(1): 33-45.

Cardinaels, E. \& Labro, E. (2008) "On the Determinants of Measurement Error in Time-Driven Costing". En: The Accounting Review, 83(3): 735-756.

Cardinaels, E. \& Van Veen-Dirks, P. (2010) "Financial versus nonfinancial information: the impact of information organization and presentation in a balanced scorecard". En: Accounting, Organizations and Society, 35: 565-578.

Chang, L., Cheng, M. \& Trotman, K.T. (2008) "The effect of framing and negotiation partner's objective on judgments about negotiated transfer prices". En: Accounting, Organizations and Society, 33(7-8): 704-717.

Dilla, W.N. \& Steinbart, P.J. (2005) "Relative weighting of common and unique balanced scorecard measures by knowledgeable decision makers". En: Behavioral Research in Accounting, 17: 43-53.

Drury, C. (2012). Management and Cost Accounting. 8th Edition. London: International Thomson Business Press.

FASB (1980). SFAC 2 concepts statement No. 2 qualitative characteristics of accounting information. Issue Date 5/1980.

Franco-Santos, M., Lucianetti, L. \& Bourne, M. (2012) "Contemporary performance measurement systems: a review of their consequences and a framework for research". En: Management Accounting Research, 23(1): 79-119.

Golman, R. \& Bhatia, S. (2012) "Performance evaluation inflation and compression". En: Accounting, Organizations and Society, 37(8): 534-543.

Griffin, R.W. \& Lopez, Y.P. (2005) "Bad Behavior in Organizations: A Review and Typology for Future Research". En: Journal of Management, 31(6): 988-1005.

Harrell, A. \& Harrison, P. (1994) "An incentive to shirk, privately held information, and managers' project evaluation decisions". En: Accounting, Organizations and Society, 19(7): 569-577.

Harwood, G.B., Pate, J.L. \& Schneider, A. (1991) "Budgeting decisions as a function of framing: an application of prospect theory's reflection effect". En: Management Accounting Research, 2(3): 161-170.

Hopwood, A.G. (1972) "An empirical study of the role of accounting data in performance evaluation". En: Journal of Accounting Research, 10: 156-182.

Ittner, C.D., Larcker, D.F. \& Meyer, M.W. (2003) "Subjectivity and the weighting of performance measures: Evidence from a balanced scorecard”. En: The Accounting Review, 78: 725-758.

Kaplan, S.E. \& Wisner, P.S. (2009) "The judgmental effects of management communications and a fifth balanced scorecard category on performance evaluation". En: Behavioral Research in Accounting, 21(2): 37-56.

Kaplan, S.E., Petersen, M.J. \& Samuels, J.A. (2012) "An Examination of the Effect of Positive and Negative Performance on the Relative Weighting of Strategically and Non-Strategically Linked Balanced Scorecard Measures". En: Behavioral Research in Accounting, 24(2): 133-151.

Libby, T., Salterio, S.E. \& Webb, A. (2004) "The balanced scorecard: the effects of assurance and process accountability on managerial judgment". En: The Accounting Review 79(4): 1075-1094. 
Liedtka, S., Church, B.K. \& Ray, M. (2008) "Performance variability, ambiguity, intolerance, and balanced scorecard-based performance assessments". En: Behavioral Research in Accounting, 20(2): 73-88.

Lipe, M.G. \& Salterio, S.E. (2000) "The balanced scorecard: judgmental effects of common and unique performance measures". En: The Accounting Review, 75(3): 283-298.

Lipe, M.G. \& Salterio, S.E. (2002) "A note on the judgmental effects of the balanced scorecard's information organization". En: Accounting, Organizations and Society, 27(6): 531-540.

Maas, V.S. \& Van Rinsum, M. (2013) "How Control System Design Influences Performance Misreporting". En: Journal of Accounting Research, 51(5): 1159-1186.

Moers, F. (2005) "Discretion and bias in performance evaluation: the impact of diversity and subjectivity". En: Accounting Organizations and Society, 30(1): 67-80.

Naranjo-Gil, D. \& Hartmann, F. (2007) "Management accounting systems, top management team heterogeneity and strategic change". En: Accounting, Organizations and Society, 32(7-8): 735-756.

Prendergast, C. \& Topel, R. (1993) "Discretion and bias in performance evaluation". En: European Economic Review, 37: 355-365.

Probst, T.M., Carneavale, P.J. \& Triandis, H.C. (1999) "Cultural Values in Intergroup and Single-Group Social Dilemmas". En: Organization Behavior and Human Decision Processes, 77(3): 171-191.

Roberts, M.L., Albright, T.L. \& Hibbets, A.R. (2004) "Debiasing balanced scorecard evaluations". En: Behavioral Research in Accounting, 16: 75-88.
Roman, F.J. (2009) "An analysis of changes to a team-based incentive plan and its effects on productivity, product quality, and absenteeism". En: Accounting, Organizations and Society, 34: 589-618.

Rowe, C. (2004) "The effect of accounting report structure and team structure on performance in cross-functional teams". En: The Accounting Review, 79(4): 1153-1180.

Schiff, A.D. \& Hoffman, L.R. (1996) "An exploration of the use of financial and nonfinancial measures of performance by executives in a service organization". En: Behavioral Research in Accounting, 8: 134-153.

Solomons, D. (1991) "Accounting and social change: A neutralist view". En: Accounting, Organizations and Society, 16(3): 287-295.

Tayler, W.B. (2010) "The balanced scorecard as a strategy-evaluation tool: the effects of implementation involvement and a causalchain focus". En: The Accounting Review, 85(3): 1095-1117.

Tinker, T. (1985). Paper prophets: A social critique of accounting. New York: Praeger Publishers Co.

Waller, W.S., Shapiro, B. \& Sevcik, G. (1999) "Do cost-based pricing biases persist in laboratory markets?". En: Accounting, Organizations and Society, 24(8): 717-739.

Waller, W.S. (1988) "Slack in participative budgeting: The joint effect of a truth-inducing pay scheme and risk preferences". En: Accounting, Organizations and Society, 13(1): 87-98.

Wong-on-Wing, B., Guo, L., Li, W. \& Yang, D. (2007) "Reducing conflict in balanced scorecard evaluations". En: Accounting Organizations and Society, 32(4-5): 363-377.

Wyatt, A.R., (1986) Standard Setting: Process and Politics, FASB Status Report No. 179 (Stamford, Conn.: FASB, 1986). 